\title{
Neuroscience today, Florence 25-27 March 2007: foreword
}

\author{
Fortunato Tito Arecchi - Alessandro Farini · \\ Riccardo Meucci · Walter G. Sannita
}

Received: 7 November 2008/Accepted: 27 November 2008/Published online: 10 January 2009

(c) Marta Olivetti Belardinelli and Springer-Verlag 2008

The emerging of the brain complex functional patterns and large-scale organization from the properties of its neuronal constituents is a major issue in today's neuroscience. Experiments and computational models indicate the structural and functional diversity of cortical neurons as a key issue in the understanding how the robustness of neuronal components combines with weak and flexible linkages between regulatory processes to account for the adaptive tunable dynamics, coding schemes and complexity of developed nervous systems. Research has focused in recent years on the communication codes among neurons, neuronal assemblies and networks; the neural properties and processes allowing resonance to occur; the mechanisms on function-related, transient neuronal synchronization; the signal/noise interaction; stochastic resonance phenomena; the information carried on by oscillatory signals of the brain, especially in the "gamma" band; etc. The variability of cortical inhibitory interneurons, interneuron networking and GABAergic sites/synapses are possibly the most conspicuous source

F. T. Arecchi

Università di Firenze, Florence, Italy

\author{
A. Farini $(\bowtie) \cdot$ R. Meucci \\ Istituto Nazionale di Ottica Applicata-CNR, Florence, Italy \\ e-mail: alessandro.farini@inoa.it \\ W. G. Sannita \\ Università di Genova, Genova, Italy \\ W. G. Sannita \\ State University of New York, Stony Brook, NY, USA
}

of neuronal diversity, also enabling virtually unlimited combinatorial effects at each time point. A close scrutiny of the available computational and non-conventional models derived from e.g. invertebrate and plant biology and their extension to higher-order neuronal operations (including "cognitive" processes) is now in order.

The international Conference "Neuroscience today: neuronal functional diversity and collective behaviors" has been planned in the framework of the European Community COST ACTION B27 "Electric neuronal oscillations and cognition (ENOC)" and took place at the Accademia dei Georgofili in Firenze (Italy), March 26-28, 2007. The main goal of the COST Action B27 (see also: http://www.manu.edu.mk/costb27) is to increase the knowledge on electric neuronal oscillations correlated to memory and attention as the basis for neuronal regulation aimed at enhancing the human performance and health. Purposes of the Conference were to focus on the integration of diverse neurons (e.g. segregated and selective for sensory input or motor organization) into networks behaving as functional assemblies, set research guidelines for the combination of (sub)cellular and system brain research, promote integrated approaches, and disseminate cultural and research programs in the framework of the COST B27 project. In this regard, the conference has provided unique opportunities to discuss new trends in neuroscience and to focus on functional neuronal diversity. Thanks are due to all the participating scientists. The available evidence and models notwithstanding, the continuum from neuronal functional diversity to collective behaviors remains to a large extent incomplete and incompletely characterized - an open question, for which no comprehensive theory appears as yet conceivable. In this respect, it is our hope that the conference and its 
proceedings will help share the relevant information on this issue and promote further research. If so, the Conference would be successful beyond the duties and purposes of a scientific event.
Thanks are due to the European Community COST ACTION B27 and Fondazione ECRF (Firenze, Italy) for financial support in the organization of the conference and publication of this special issue. 